Research

\title{
Sequence diversity and natural selection at domain I of the apical membrane antigen I among Indian Plasmodium falciparum
} populations

\author{
Sheena Garg11, Mohammad T Alam¹, Manoj K Das², Vas Dev³, \\ Ashwani Kumar ${ }^{4}$, Aditya P Dash ${ }^{5}$ and Yagya D Sharma*1
}

Address: ${ }^{1}$ Department of Biotechnology, All India Institute of Medical Sciences, Ansari Nagar, New Delhi, India, ${ }^{2}$ National Institute of Malaria Research (Field Station), Car Nicobar, Andaman and Nicobar Islands, India, ${ }^{3}$ National Institute of Malaria Research (Field Station), Kamrup, Assam, India, ${ }^{4}$ National Institute of Malaria Research (Field Station), Goa, India and ${ }^{5}$ National Institute of Malaria Research, Delhi, India

Email: Sheena Garg - sheenagarg2000@yahoo.com; Mohammad T Alam - taiims@email.com; Manoj K Das - manojdas2003@yahoo.co.in; Vas Dev - mrcassam@hotmail.com; Ashwani Kumar - ashwani_07@yahoo.com; Aditya P Dash - apdash@gmail.com;

Yagya D Sharma* - ydsharma_aiims@yahoo.com

* Corresponding author

Published: 22 November 2007

Malaria Journal 2007, 6:154 doi:10.1186/1475-2875-6-154
Received: 3 August 2007

Accepted: 22 November 2007

This article is available from: http://www.malariajournal.com/content/6/I/154

(c) 2007 Garg et al; licensee BioMed Central Ltd.

This is an Open Access article distributed under the terms of the Creative Commons Attribution License (http://creativecommons.org/licenses/by/2.0), which permits unrestricted use, distribution, and reproduction in any medium, provided the original work is properly cited.

\begin{abstract}
Background: The Plasmodium falciparum apical membrane antigen I (AMAI) is a leading malaria vaccine candidate antigen. The complete AMAI protein is comprised of three domains where domain I exhibits high sequence polymorphism and is thus named as the hyper-variable region (HVR). The present study describes the extent of genetic polymorphism and natural selection at domain I of the amal gene among Indian P. falciparum isolates.
\end{abstract}

Methods: The part of the amal gene covering domain I was PCR amplified and sequenced from 157 P. falciparum isolates collected from five different geographical regions of India. Statistical and phylogenetic analyses of the sequences were done using DnaSP ver. 4. 10. 9 and MEGA version 3.0 packages.

Results: A total of 57 AMAI haplotypes were observed among 157 isolates sequenced. Forty-six of these 57 haplotypes are being reported here for the first time. The parasites collected from the high malaria transmission areas (Assam, Orissa, and Andaman and Nicobar Islands) showed more haplotypes $(H)$ and nucleotide diversity $\pi$ as compared to low malaria transmission areas (Uttar Pradesh and Goa). The comparison of all five Indian P. falciparum subpopulations indicated moderate level of genetic differentiation and limited gene flow (Fixation index ranging from 0.048 to 0.13 ) between populations. The difference between rates of non-synonymous and synonymous mutations, Tajima's D and McDonald-Kreitman test statistics suggested that the diversity at domain I of the AMAI antigen is due to positive natural selection. The minimum recombination events were also high indicating the possible role of recombination in generating AMAI allelic diversity.

Conclusion: The level of genetic diversity and diversifying selection were higher in Assam, Orissa, and Andaman and Nicobar Islands populations as compared to Uttar Pradesh and Goa. The amounts of gene flow among these populations were moderate. The data reported here will be valuable for the development of AMAI-based malaria vaccine. 


\section{Background}

The Plasmodium falciparum malaria is one of the major causes of morbidity and mortality in tropical and subtropical countries. Worldwide, approximately 300-500 million people are affected each year with the disease and children below 5 years of age and pregnant women are the largest target groups. The global burden of human malaria is continued to increase due to lack of an effective malaria vaccine, development of insecticide resistance in the Anopheles, and drug resistance in the parasite. Several parasite molecules have been tested for their potential as a vaccine candidate antigens [1]. Majority of these antigens are expressed on the parasite's surface or the parasitized erythrocytes. The Plasmodium proteins expressed on the parasite's surface are more exposed to human immune system and thus have been found to exhibit high antigenic diversity [2]. Genetic polymorphisms in the parasite antigens help them to evade the host's protective immune response but at the same time it hampers the development of an effective vaccine which can contain this disease.

The $P$. falciparum apical membrane antigen 1 (AMA1) is an $83 \mathrm{kDa}$ membrane protein expressed in the late schizont stage of the parasite [3,4]. Although the exact function of AMA1 is not known, its role in merozoite invasion has been documented by several investigators and antibodies raised against this protein have been shown to block invasion of the parasites into human RBCs [5-9]. Natural immune responses (both humoral and cellular) against the AMA1 antigen have been observed in populations exposed to P. falciparum malaria [10]. Thus, AMA1 has been included as one of the potential components of an asexual stage multivalent malaria vaccine. An AMA1based malaria vaccine has recently been tested in phase I clinical trial [11]. Although AMA1 is less polymorphic than other sporozoite or merozoite proteins, some extent of variation are present across the entire AMA1 sequence [12-14] but the rate of non-synonymous ( $\mathrm{dN}$ ) mutations at domain I (aa 138-308) has always been higher indicating that this domain is under strong diversifying selection $[13,15,16]$.

Antigenic variation in Plasmodium has hampered malaria vaccine development because the antibodies elicited against one allele of an antigen might not necessarily inhibit RBCs invasion by the parasites expressing different alleles of that antigen $[17,18]$. Therefore, polymorphism studies on the AMA1 antigen of natural $P$. falciparum populations need to be done before initiating any clinical trials of a vaccine using this antigen. This study describes the extent of antigenic diversity and natural selection at domain I of AMA1 among Indian P. falciparum populations. The results obtained here will have significant implications in malaria vaccine development.

\section{Methods \\ Parasite collection}

Patients attending malaria clinics at Kamprup (Assam), Cuttack (Orissa), Car Nicobar (A \& N), Ghaziabad and Aligarh (UP) and Panjim (Goa) were tested for the presence of P. falciparum. About 50-100 $\mu$ l of blood was collected from the microscopically $P$. falciparum positive patients with their informed consent. The institutional ethical guidelines were followed for blood collection.

\section{DNA isolation and PCR amplification of the ama I gene}

DNA from $P$. falciparum infected blood was isolated as described previously [19]. This DNA was used to amplify the complete $2 \mathrm{~kb}$ ama1 gene using the AMA1-F (5'ACAAAAATGAGAAAATTATACTGC-3') and AMA1-R (5'TTTTAATAGTATGGTTTTTCCATC-3') primers. The cycling parameters for the primary PCR were as follows: $10 \mathrm{~min}$ utes initial denaturation at $94^{\circ} \mathrm{C}$ followed by 35 cycles with 1 minute denaturation at $94^{\circ} \mathrm{C}, 1$ minute annealing at $55^{\circ} \mathrm{C}, 2$ minute extension at $72^{\circ} \mathrm{C}$ and a final 10 minute extension at $72^{\circ} \mathrm{C}$. The primary PCR product was diluted 10-times and $2 \mu \mathrm{l}$ of it was used in nested-PCR to amplify the $500 \mathrm{bp}$ region encompassing domain I (or hyper-variable region-HVR) using the AHVR-F (5'CTGGAACTCAATATAGACTTC-3') and AHVR-R (5'TTCTTTCTAGGGCAAACTTTTTC-3') primers. The cycling parameters for nested primers were the same as for primary PCR primers except that the extension at $72^{\circ} \mathrm{C}$ was carried out for 1 minute.

\section{Nucleotide sequencing and sequence analysis}

The desired 500 bp DNA band was excised from the agarose gel and purified as described earlier [19]. Sequencing of the product was done from both strands using AHVR-F and AHVR-R primers. Sequencing PCR cycling parameters and other downstream protocols for sequencing were same as described earlier [19]. The sequences were edited and the translated amino acids were aligned amongst themselves and also with P. falciparum 3D7 [GenBank; NC 004315], Dd2 [GenBank; AASM01002002] and HB3 [GenBank; AANS01001407] strains AMA1 sequences using GeneDoc Version 2.6.002 program [20]. In addition, BLAST searches were also performed to compare these AMA1 haplotypes with the other AMA1 sequences available in the GenBank database. The sequences reported in this paper have been deposited in the database [GenBank; EF413088-EF413170].

\section{Statistical and phylogenetic analyses}

The numbers of segregating sites (S), observed nucleotide diversity per site between any two sequences assuming that the sample is random $(\pi)$, number of haplotypes $(H)$, haplotype diversity (Hd), and average number of pairwise nucleotide differences within population $(\mathrm{K})$, linkage disequilibrium (LD) and recombination parameters ( $R$ and 
$\mathrm{Rm})$ were calculated using DnaSP ver. 4. 10. 9 [21]. The $\pi$ diversity was also calculated on sliding window of 100 bases, with a step size of $25 \mathrm{bp}$ in order to estimate the step-wise diversity across domain I. The rates of synonymous (dS) and non-synonymous $(\mathrm{dN})$ mutations were computed on MEGA version 3.0 [22] using the method of Nei and Gojobori's [23] with the Jukes and Cantor (JC) correction. The $\mathrm{dN}$-dS difference and Tajima's D [24] test statistics were applied to test the neutral theory of evolution. Positive values for Nei-Gojobori $(\mathrm{dN}-\mathrm{dS})$ and Tajima's D correspond to positive natural selection whereas negative values correspond to negative or purifying selection [25]. In addition, McDonald \& Kreitman (MK) [26] test was also applied as a test of neutrality taking partial $P$. reichenowi AMA1 sequence as an outgroup using DnaSP. The MK test compares dS and dN mutations within and between species. Under neutrality, the ratio of $\mathrm{dN} / \mathrm{dS}$ mutations between species should be the same as the ratio of $\mathrm{dN} / \mathrm{dS}$ mutations within species. However, when the $\mathrm{dN} / \mathrm{dS}$ ratio is greater between species than within species, it is said to be under positive natural selection. In case of negative selection, the $\mathrm{dN} / \mathrm{dS}$ ratio between species is lower than within species. The genetic differentiation among the parasite populations was calculated in terms of fixation index (Fst) that estimates diversity within a subpopulation with respect to total genetic diversity. In addition, average number of pairwise nucleotide differences (Kxy), nucleotide substitution per site (Dxy), and net nucleotide substitution per site (Da) between populations were also calculated. The above parameters were also estimated on DnaSP. Phylogenetic analysis was performed by neighbour-joining (NJ) method with Kimura 2-parameter distance matrix [27] in MEGA version 3.0 [22].

\section{Results}

Haplotype diversity and amino acid changes at domain I

The nucleotide sequence encoding domain I of the AMA1 was determined from 157 clinical isolates collected from five different geographical regions (Assam, $\mathrm{n}=28$; Orissa, $\mathrm{n}=35 ; \mathrm{A} \& \mathrm{~N}, \mathrm{n}=40$; UP, $\mathrm{n}=36$; and Goa, $\mathrm{n}=18$ ) of India with different malaria transmission intensities. The analysis of the $456 \mathrm{bp}$ sequence obtained (corresponding to $448-903 \mathrm{bp}$ region of the ama1 gene encoding for amino acid residues $150-301$ ) for these 157 isolates revealed that, 418 nucleotide positions were either monomorphic or invariable. Remaining $44(9.64 \%)$ sites were polymorphic ( 8 singleton variables and 36 parsimony informative), that generated 57 different AMA1 haplotypes (H1 to H57) among Indian P. falciparum populations (Additional File 1). The two predominant AMA1 haplotypes were H56 $(13.37 \%, \mathrm{n}=157)$ and H57 $(15.92 \%, \mathrm{n}=157)$ (Additional File 1). Thirty-one haplotypes (H1 - H31) were unique as each one of them was found in a single isolate only. The BLAST search against all
P. falciparum AMA1 sequences available in the GenBank database confirmed that 46 of these 57 haplotypes were new as they have not been reported earlier from any other region of the world. The remaining eleven haplotypes showed $100 \%$ identity with the AMA1 sequences reported from other countries $[12,13,16,28]$. The H56 was the only haplotype that showed $100 \%$ identity with the already reported AMA1 sequences [AAG50135] from Indian P. falciparum isolate [13]. Furthermore, none of the isolates had identical sequences to the $3 \mathrm{D} 7, \mathrm{Dd} 2$ or HB3 AMA1 alleles.

The number of AMA1 haplotypes in Assam $(\mathrm{H}=20)$, Orissa $(H=23)$, and $A \& N(H=18)$ were higher than those seen in UP $(H=10)$ and Goa $(H=7)$. Majority $(14.28 \%, \mathrm{n}=28)$ of the isolates from Assam showed H48 haplotype which was not found among the isolates sequenced from the four other areas. Similarly, H57 was the predominant haplotype in Orissa and A \& N but not seen in Assam (Additional File 2). Certain haplotypes were found to be region specific, and also none of the 57 haplotypes was common to all the five study areas (Additional File 2). Most $(47.22 \%, \mathrm{n}=36)$ of the isolates from UP had H56 AMA1 haplotype that was not found in Goa isolates.

Amino acid polymorphisms were observed at 29 of the 152 codons (456 bp) across the entire domain I (Additional File 1). Of these 29 polymorphic sites, 17 [codons$162(\mathrm{~N} / \mathrm{K}), 167$ (T/K), $173(\mathrm{~N} / \mathrm{K}), 175(\mathrm{D} / \mathrm{Y}), 189(\mathrm{~L} / \mathrm{P})$, $190(\mathrm{M} / \mathrm{I}), 196(\mathrm{D} / \mathrm{N}), 199(\mathrm{R} / \mathrm{K}), 206(\mathrm{~K} / \mathrm{E}), 218(\mathrm{~S} / \mathrm{P})$, $225(\mathrm{~N} / \mathrm{I}), 228(\mathrm{~N} / \mathrm{K}), 242(\mathrm{Y} / \mathrm{D}), 244(\mathrm{D} / \mathrm{N}), 267$ (E/Q), $269(\mathrm{~K} / \mathrm{I})$, and $296(\mathrm{D} / \mathrm{H})$ ] showed di-morphic, 8 [codons $172(\mathrm{G} / \mathrm{E} / \mathrm{V}), 204(\mathrm{~N} / \mathrm{D} / \mathrm{G}), 207(\mathrm{Y} / \mathrm{D} / \mathrm{V}), 230(\mathrm{~K} / \mathrm{E} / \mathrm{Q})$, $243(\mathrm{~N} / \mathrm{K} / \mathrm{E}), 282(\mathrm{~K} / \mathrm{I} / \mathrm{N}), 283(\mathrm{~S} / \mathrm{L} / \mathrm{P})$ and $285(\mathrm{Q} / \mathrm{E} / \mathrm{R})]$ showed tri-morphic, 3 [codons 187 (N/E/K/D), 200 (D/L/ $\mathrm{H} / \mathrm{R})$ and $201(\mathrm{~F} / \mathrm{L} / \mathrm{S} / \mathrm{V})]$ showed tetra-morphic and only one site [codon $197(\mathrm{G} / \mathrm{D} / \mathrm{H} / \mathrm{Q} / \mathrm{V})$ ] showed penta-morphic alleles.

The number of polymorphic codons and number of total mutations for Assam (25 polymorphic codons and 37 mutations), Orissa (27 polymorphic codons and 38 mutations) and A \& N (24 polymorphic codons and 33 mutations) isolates were almost equal but greater than the isolates from UP (20 polymorphic codons and 26 mutations) and Goa (16 polymorphic codons and 20 mutations). Sixteen of the 29 polymorphic codons were common to all the five regions whereas, remaining 13 codons were showing region-specific polymorphisms. The N173K, L189P, and D244N mutations were found among the isolates from Assam and Orissa only but not among A \& N, UP and Goa isolates. The codon polymorphisms D175Y, D/N204G, Y/D207V, S218P and Q/ E285R were $A$ \& $N$ specific whereas $G / E 172 V, E / N /$ K187D, R199K and K269I were Orissa-specific. Only two 
Assam-specific mutations were observed (K/I282N and S/ L283P) while no region-specific mutations was found among the isolates sequenced from UP and Goa.

\section{Nucleotide diversity and genetic differentiation}

The haplotype (gene) diversity (Hd) for all 157 sequences was calculated to be $0.948 \pm 0.010 \mathrm{SD}$. The average number of pairwise nucleotide differences within Indian P. falciparum population (K) was 10.13 with the overall $\pi$ diversity $0.0222 \pm 0.0005 \mathrm{SD}$ (Table 1 ). However, the sliding window analysis of the entire $456 \mathrm{bp}$ region estimated $\pi$ diversity ranging from $0.00668-0.05126$ with the highest value between nucleotide positions 50 and 200 (0.01501 to 0.02488 ) (Figure 1$)$. A total of 12 minimum number of recombination $(\mathrm{Rm})$ events were observed between adjacent polymorphic sites (Table 2). The estimates of $\mathrm{R}=4 \mathrm{Nr}$ between adjacent sites and per gene were 0.123 and 56.2 respectively. The haplotype diversity (Hd), nucleotide diversity per site $(\pi)$ and average number of pairwise nucleotide differences within population $(\mathrm{K})$ showed regional variations (Table 1 ). The $\pi$ diversity was highest between nucleotide positions 50 and 200 among all five populations as estimated by sliding window method using a step size of $25 \mathrm{bp}$ (Figure 1).

Inter-population nucleotide differences (Kxy) varied from 8.3 (between UP and Goa) to 11.53 (Assam and A \& N) (Table 3). Similarly, the average number of nucleotide (Dxy) and net nucleotide (Da) substitutions per site between populations ranged from 0.018 to 0.025 and 0.00135 to 0.0755 respectively (Table 3 ). Although Fst statistics for the whole Indian P. falciparum populations was found to be 0.085 , the Fst values between different geographical populations varied from 0.048 (between Orissa and Goa) to 0.133 (between UP and Orissa) (Table 3 ). The phylogenetic analysis of the sequences revealed that only 14 AMA1 alleles were shared by more than one geographical area (Additional File 2). Therefore, no region-wise clustering of the alleles was observed (Figure 2 ). Inter-population comparison of the total (TM) versus shared (SM) number of mutations also showed similar

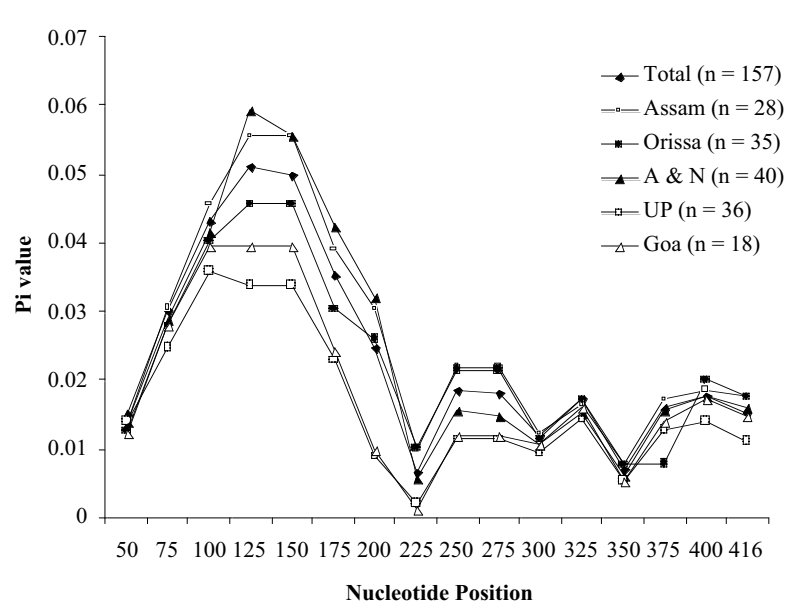

\section{Figure I}

Sliding window plot of the nucleotide diversity per site $(\pi)$ comparing the level of genetic diversity among the domain I sequences of the parasite from all five study areas. The $\pi$ values were calculated on DnaSP with window length 100 bp and step size of $25 \mathrm{bp}$. In all study areas, the maximum diversity was seen between the nucleotide positions 50 and 200 bps. $n$; number of $P$. falciparum isolates.

pattern of genetic differentiation as maximum number of shared mutations were found between Orissa \& Goa (19 of 41 mutations were shared), and Goa and A \& N (18 of 37 mutations were shared) (Table 3 ). Thus the data on phylogeny and Fst indicated moderate level of genetic differentiation and thus a limited movement of genes between populations.

\section{Evidence of selection and recombination}

The average difference of $\mathrm{dN}-\mathrm{dS}$ for all 157 AMA1 sequences was $0.027 \pm 0.006 \mathrm{SD}$, indicating that domain I is under positive natural selection (Table 1). The $\mathrm{dN}-\mathrm{dS}$ differences were almost equal for Assam, Orissa and A \& $\mathrm{N}$ populations but were greater than those observed for

Table I: Measures of DNA sequence polymorphisms and tests of neutrality at domain I of AMA I among Indian P. falciparum populations

\begin{tabular}{|c|c|c|c|c|c|c|c|c|c|c|}
\hline $\begin{array}{c}\text { Study areas } \\
(\mathrm{n}=157)\end{array}$ & $\begin{array}{c}\text { Segregating } \\
\text { sites (S) }\end{array}$ & $\begin{array}{c}\text { Singleton } \\
\text { variable sites }\end{array}$ & $\begin{array}{c}\text { Parsimony } \\
\text { informative sites }\end{array}$ & $\begin{array}{l}\text { Total no. of } \\
\text { mutations }\end{array}$ & $\mathbf{K}$ & $\mathbf{H}$ & Hd \pm S.D & $\pi \pm \mathbf{S . D}$ & dN-dS $\pm S . E$ & Tajima's D \\
\hline Assam $(n=28)$ & 34 & 6 & 28 & 38 & 10.97 & 20 & $0.971 \pm 0.018$ & $0.0240 \pm 0.0009$ & $0.029 \pm 0.006$ & 0.461 \\
\hline Orissa $(n=35)$ & 35 & 5 & 30 & 40 & 9.96 & 23 & $0.948 \pm 0.025$ & $0.0218 \pm 0.0015$ & $0.027 \pm 0.005$ & 0.094 \\
\hline$A \& N(n=40)$ & 34 & 6 & 28 & 35 & 10.34 & 18 & $0.926 \pm 0.022$ & $0.0226 \pm 0.0008$ & $0.027 \pm 0.007$ & 0.895 \\
\hline UP $(n=36)$ & 24 & 6 & 18 & 26 & 7.31 & 10 & $0.748 \pm 0.066$ & $0.0160 \pm 0.0014$ & $0.020 \pm 0.005$ & 0.576 \\
\hline Goa $(n=18)$ & 19 & I & 18 & 20 & 8.07 & 7 & $0.882 \pm 0.039$ & $0.0177 \pm 0.0011$ & $0.023 \pm 0.006$ & 1.518 \\
\hline Total $(n=157)$ & 44 & 8 & 36 & & 10.13 & 57 & $0.948 \pm 0.010$ & $0.0222 \pm 0.0005$ & $0.027 \pm 0.006$ & 0.429 \\
\hline
\end{tabular}

n; number of isolates, S; Number of segregating (polymorphic/variable) sites; K; Average number of pairwise nucleotide differences, $\mathbf{H}$; Number of haplotypes, Hd; Haplotype diversity; $\pi$; Observed average pairwise nucleotide diversity; dN-dS; rate of non-synonymous mutations minus rate of synonymous mutations; D; Tajima's D test statistics. 
Table 2: Comparison of different estimates of recombination events between all five study areas

\begin{tabular}{llll}
\hline Regions & $\mathbf{R}^{\mathbf{a}}$ & $\mathbf{R}^{\mathbf{b}}$ & $\mathbf{R m}$ \\
\hline Assam $(\mathrm{n}=28)$ & 0.178 & 81 & 9 \\
Orissa $(\mathrm{n}=35)$ & 0.118 & 53.7 & 11 \\
A \& N $(\mathrm{n}=40)$ & 0.057 & 26 & 7 \\
UP $(\mathrm{n}=36)$ & 0.016 & 7.5 & 8 \\
Goa $(\mathrm{n}=18)$ & 0.074 & 33.7 & 6 \\
\hline Total $(\mathrm{n}=157)$ & 0.123 & 56.2 & 12 \\
\hline
\end{tabular}

Note: The $\mathrm{R}$ and $\mathrm{Rm}$ were estimated excluding the sites containing alignment gaps or those segregating for three nucleotides. The $R$ was computed using $R=4 \mathrm{Nr}$, where $N$ is the population size and $r$ is the recombination rate per sequence (per gene) $n$, number of isolates; $\mathbf{R}^{\mathbf{a}}$, recombination parameter between adjacent sites; $\mathbf{R}^{\mathrm{b}}$, recombination parameter for entire gene; $\mathbf{R m}$, minimum number of recombination events between adjacent sites

UP and Goa parasite populations (Table 1). The positive value 0.429 for Tajima's D also indicated that positive natural selection at domain I might be the reason for the increased allelic diversity observed (Table 1). Similar results were obtained when the McDonald \& Kreitman (MK) test was applied to this data. The MK test showed more non-synonymous $(\mathrm{dN})$ mutations within species (dN 36 vs. dS 3 ) and fixed difference between species ( $d N$ 7 vs. dS 4) indicating positive natural selection (Fisher's exact test $\mathrm{P}=0.033, \mathrm{P}>0.01$; Neutrality index $=6.85 ; \alpha=$ $-5.85)$.

The minimum number of recombination events (Rm) between adjacent polymorphic sites for Assam, Orissa, A $\&$ N, UP and Goa isolates were 9, 11, 7, 8 and 6 respectively (Table 2 ). The value of $\mathrm{R}$ (both between adjacent sites and for the entire domain) was highest in Assam (0.178 and 81$)$, followed by Orissa ( 0.118 and 53.7$)$, Goa (0.0741 and 33.3), A \& N (0.0571 and 26), and UP (0.0165 and 8) parasite population. The higher value of the recombination parameters ( $\mathrm{Rm}$ and $\mathrm{R}$ ) indicate that high meiotic recombination is taking place between the sites generating genetic diversity in the gene. As LD index $\mathrm{R}^{\wedge 2}$ plotted against the nucleotide distances also demonstrated a decline across the entire 456 bp region (Figure 3 ), it can be assumed that intragenic recombination may be contributing to the increased diversity observed at AMA1 domain I.

\section{Discussion}

Antigenic variation in the natural $P$. falciparum populations is one of the major obstacles in the development of an effective vaccine against malaria [1,29]. A vaccine based on one allelic form of an antigen might not provide full protective immunity against the heterologous $P$. falciparum strains, carrying other alleles of that antigen [30].
Therefore, multiple allelic forms of an antigen may need to be incorporated in a vaccine to make it more efficacious against any natural $P$. falciparum infection [13,31]. In this regard it becomes absolutely essential to investigate the antigenic repertoires of the $P$. falciparum populations from different malaria endemic regions. The complete AMA1 protein is comprised of three distinct domains [32]. This study includes only domain I as the rate of non-synonymous mutations at this domain has always been found to be higher due to the phenomenon of positive natural selection [13-16,28]. A total of 57 AMA1 haplotypes were observed from $157 \mathrm{P}$. falciparum isolates studied from five different regions of India. The $\mathrm{H} 56$ and $\mathrm{H} 57$ were the predominant haplotypes (Additional File 1). All measures of genetic polymorphisms showed regional variation among these five Indian P. falciparum populations (Table 1). The extent of genetic diversity depends on the malaria transmission intensity and the prevalence of malaria parasite in that region. The extent of genetic polymorphisms at domain I was higher amongst the isolates from Assam, Orissa and A \& N as compared to those from UP and Goa isolates (Figure 1, Table 1). It is important to note that Assam, Orissa and A \& N are considered as high malaria transmission areas whereas UP and Goa are low malaria transmission areas of India [33].

Analysis of the AMA1 sequences indicated that the level of diversity amongst Indian $P$. falciparum isolates was relatively lower than the reported diversity for African isolates but was quite similar to the Asian and South American populations $[13,16,28]$. A total of 35 AMA1 alleles (with $\pi$ diversity 0.027 at domain I) were found when 51 Nigerian $P$. falciparum isolates were sequenced, while only 18 alleles (with $\pi$ diversity 0.025 at domain I) were found when an almost equal number $(n=50)$ of isolates were sequenced from Thailand [16,28]. In another study, a total of 27 AMA1 alleles were found when 168 isolates (with $\pi$ diversity 0.026 at domain I) were analysed from PNG [12]. It is important to note that number of polymorphic AMA1 codons among PNG [12] isolates (29 codons, 168 isolates) were similar to those observed amongst the Indian isolates (this study, 29 codons, 157 isolates). Although the number of haplotypes among Indian isolates $(\mathrm{H}=57)$ were greater than those reported for the PNG isolates $(\mathrm{H}=27)$ however, the $\pi$ diversity was higher for PNG $(\pi=0.026)$ as compared to Indian isolates $(\pi=0.022)$.

Only 11 of the 57 haplotypes were identical to some of the AMA1 haplotypes observed in Thai, PNG and Nigerian $P$. falciparum isolates $[12,13,16,28]$. The $\mathrm{H} 56$ was the only haplotype which exhibited $100 \%$ identity with previously reported AMA1 alleles [AAG50135] from Indian isolate [13]. On the other hand 46 AMA1 haplotypes have been reported here for the first time. 


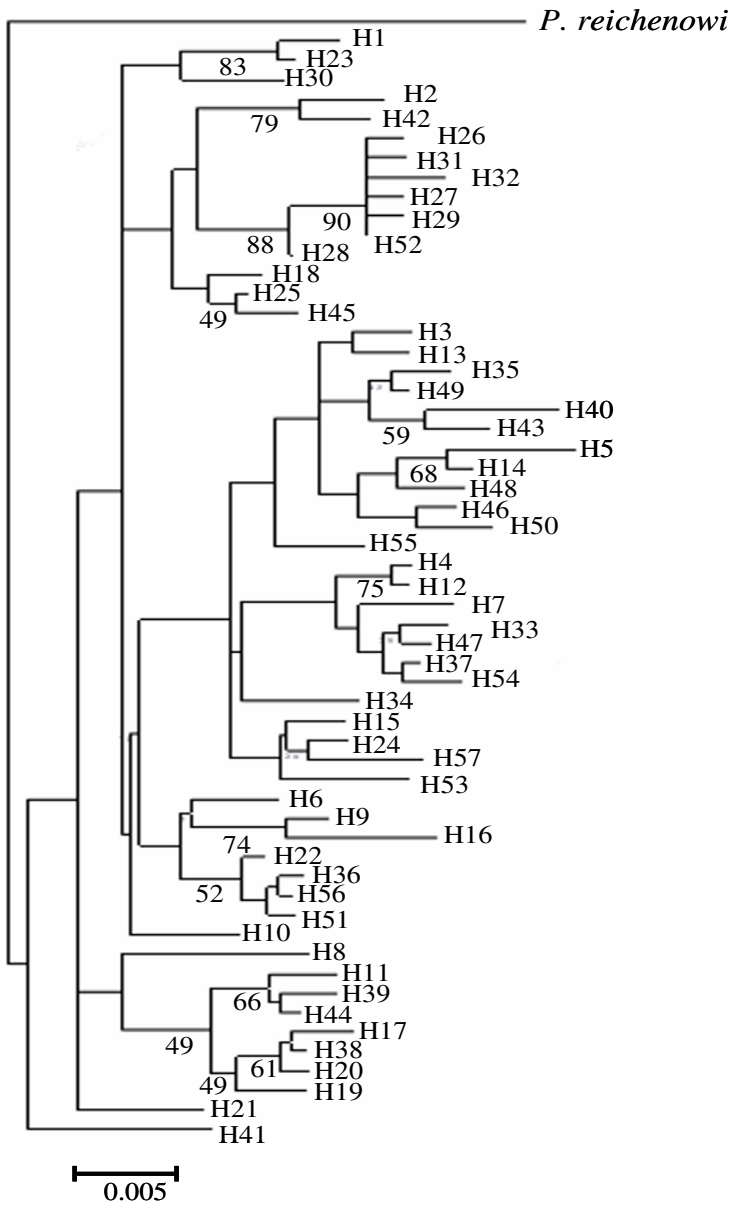

\section{Figure 2}

A neighbor-joining $(\mathrm{NJ})$ tree depicting the relationships between different AMAI haplotypes observed among Indian $P$. falciparum populations. The distance matrix was prepared using Kimura 2-parameter evolutionary model. Alignment substitutions were considered for analysis while gaps were ignored. Numbers below the line indicate percentage bootstrap values for 1000 replications. The scale bar represents a genetic distance. The partial AMAI sequence of $P$. reichenowi [A]252087], the closet species to $P$. falciparum was taken as an out group [35].

Table 3: Inter-population genetic differentiation of the parasites

\begin{tabular}{cccccccc}
\hline S. No. & Population-I & Population-2 & Kxy & Dxy & Dac $^{\mathbf{b}}$ & Fst $^{\mathbf{d}}$ & TM/SMe $^{\mathbf{c}}$ \\
\hline I & Assam & UP & 10.087 & 0.02212 & 0.00207 & 0.09339 \\
2 & Assam & Orissa & 11.273 & 0.02472 & 0.00176 & 0.07101 & $38 / 26$ \\
3 & Assam & Goa & 10.764 & 0.02361 & 0.00271 & 0.11488 & $38 / 35$ \\
4 & Assam & AN & 11.531 & 0.02529 & 0.00191 & 0.07551 & $45 / 28$ \\
5 & UP & Orissa & 9.977 & 0.02188 & 0.00293 & 0.13380 & $41 / 25$ \\
6 & UP & Goa & 8.313 & 0.01823 & 0.00135 & 0.07421 & $26 / 20$ \\
7 & UP & AN & 9.909 & 0.02173 & 0.00237 & 0.10894 & $37 / 24$ \\
8 & Orissa & Goa & 9.481 & 0.02079 & 0.00100 & 0.04819 & $41 / 19$ \\
9 & Orissa & AN & 10.983 & 0.02409 & 0.00181 & 0.07517 & $47 / 28$ \\
\hline 10 & Goa & AN & 9.739 & 0.02136 & 0.00116 & 0.05414 & $37 / 18$ \\
\hline
\end{tabular}

a; Average number of nucleotide differences between populations

b; The average number of nucleotide substitutions per site between populations,

c; The number of net nucleotide substitutions per site between populations,

d; Fixation index, a measure of genetic differentiation between populations (range from 0 to +1 ),

e, TM, total number of mutations in both populations; SM, Numbers of mutations shared between populations 


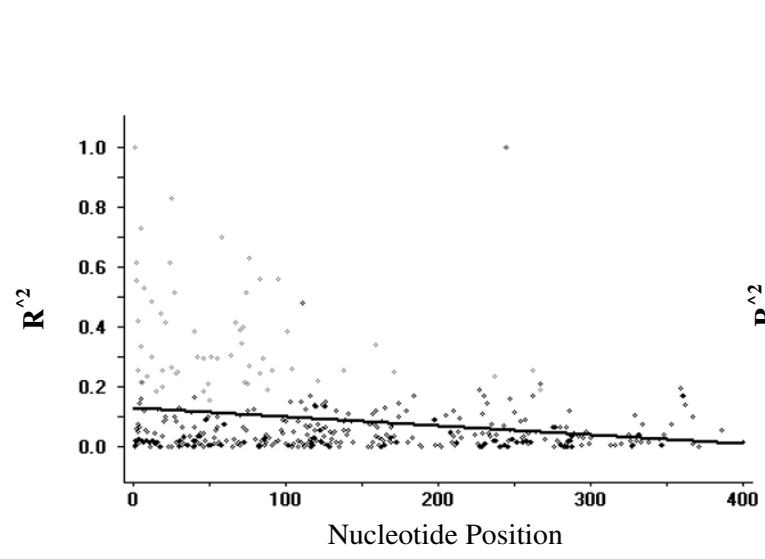

A

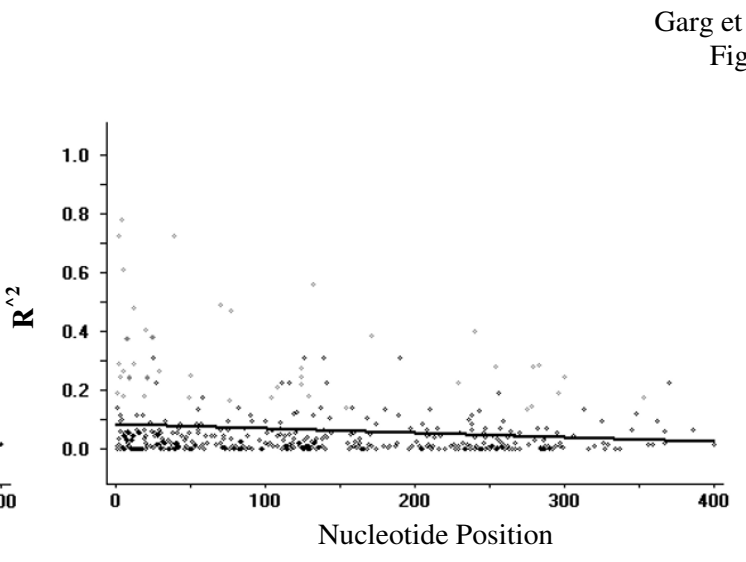

B

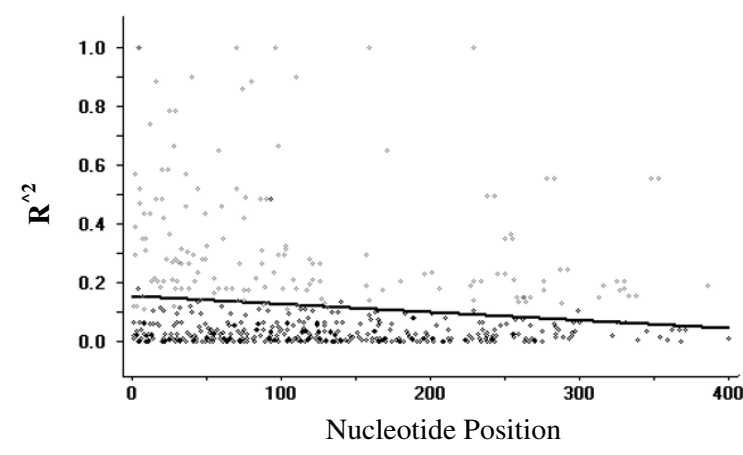

$\mathbf{C}$

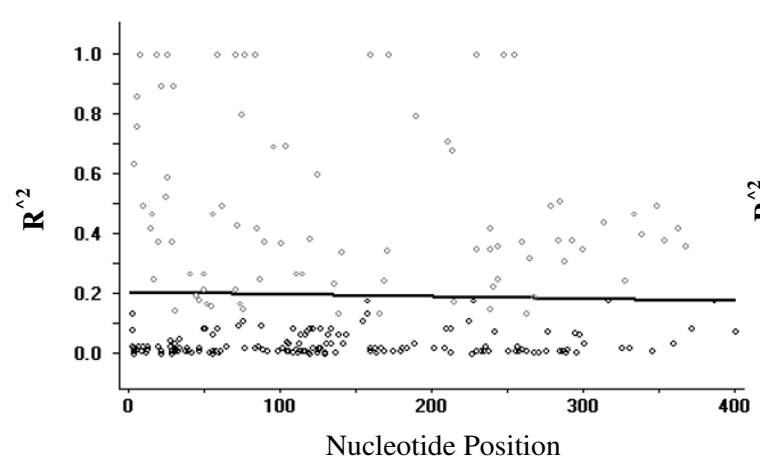

$\mathbf{E}$

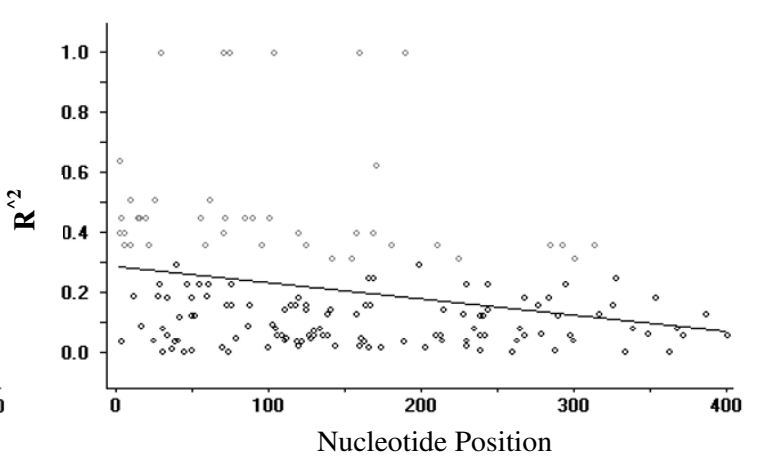

D

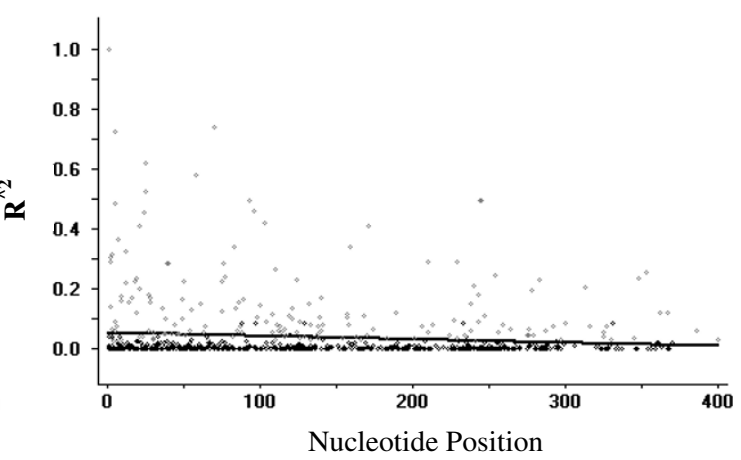

$\mathbf{F}$

\section{Figure 3}

The linkage disequilibrium (LD) plot showing non-random association between nucleotide variants at different polymorphic sites. The $R^{\wedge 2}$ values are plotted against the nucleotide distances with two-tailed Fisher's exact test of significance using DnaSP. The value of $L D$ index (range from $-I$ to $+I$ ) declines with increasing nucleotide distance, indicating that recombination events are taking place. The analysis was performed considering all polymorphic sites. (A) Assam, (B) Orissa, (C) A \& N, (D) Goa, (E) UP, and (F) Total. 
Although polymorphisms were observed at 29 of the 152 codons of domain I, majority (17 of 29) of them were showing dimorphic amino acids which is in accordance with previous studies $[12,13]$. Only 5 alternative amino acid residues $(\mathrm{G} / \mathrm{D} / \mathrm{H} / \mathrm{Q} / \mathrm{V})$ were present in the Indian parasite population at the highly polymorphic codon 197 of domain I as compared to 7 alternative substitutions reported from elsewhere [34]. A pdb structure of AMA1 using all these codon polymorphisms was also in accordance with previous studies [9]. Thus any vaccine developed using the AMA1 antigen will need to take into account all these variations observed within the gene.

The overall difference between $\mathrm{dN}$ and $\mathrm{dS}$ was positive ( $\mathrm{dN}>\mathrm{dS}$ ) suggesting that domain I is under positive natural selection [25]. Similar results were obtained when Tajima's D and McDonald \& Kreitman (MK) test were applied. Enormous evidence now supports the theory that the high allelic diversity at domain I is due to strong selection pressure by host immune response [16,17,25,34]. The estimates of inter-population comparisons (Kxy, Dxy, $\mathrm{Da}, \mathrm{TM} / \mathrm{TS}$ and Fst) supported moderate levels of genetic differentiation amongst the Indian populations which are relatively higher than the Fst values between Wosera \& Nigerian (Fst $=0.0133)$, and Thai \& Nigerian $(F s t=0.033)$ populations (Table 3 ) $[12,13,28]$.

\section{Conclusion}

In conclusion, the present study shows that domain I of AMA1 among Indian P. falciparum isolates exhibits fairly high allelic diversity that differs from one region to another. The regional differences in AMA1 allelic diversity may pose a problem for a vaccine based on this antigen. Results obtained in this study also demonstrate that polymorphisms at domain I is due to positive natural selection. Furthermore, moderate level of gene flow was observed amongst these parasite populations. This data on AMA1 polymorphism from Indian $P$. falciparum isolates will be valuable for the development of AMA1-based malaria vaccine.

\section{List of abbreviations}

AMA1, apical membrane antigen; UP, Uttar Pradesh; A \& $\mathrm{N}$, Andaman and Nicobar Islands.

\section{Authors' contributions}

SG performed PCR and sequencing. MTA analysed the sequences, performed statistical and phylogenetic analyses and drafted the manuscript. MKD, VD, AK, APD participated in the design of the study. YDS conceived of the study, and participated in its design and coordination, analysed the data and also drafted the manuscript. All authors read and approved the final manuscript.

\section{Additional material}

\section{Additional file 1}

Amino acid polymorphisms at domain I of the P. falciparum AMA1 among Indian isolates. The data provided represent the occurrence of codon polymorphisms at AMA1 domain I among Indian P. falciparum isolates.

Click here for file

[http://www.biomedcentral.com/content/supplementary/14752875-6-154-S1.pdf]

\section{Additional file 2}

Region-wise distribution and allelic frequencies of the AMA1 haplotypes. The data provided represent the region-wise distribution and frequencies of the AMA1 haplotypes found in the five study areas of India.

Click here for file

[http://www.biomedcentral.com/content/supplementary/14752875-6-154-S2.pdf]

\section{Acknowledgements}

We thank Dr. Ananias Escalante for his intellectual input while writing this manuscript. We also thank Ms. Sumiti Vinayak and Dr. Misbah Siddiqui for proofreading the manuscript. SG and MTA acknowledge Department of Biotechnology (DBT), and Council of Scientific and Industrial Research (CSIR) for Senior Research Fellowships, respectively. Financial support for the work granted by Indian Council of Medical Research (ICMR), and Department of Biotechnology (DBT), Government of India, is acknowledged. We are also grateful to Bioinformatics facilities of Biotechnology Information System (BTIS).

\section{References}

I. Genton B, Reed $\mathrm{ZH}$ : Asexual blood-stage malaria vaccine development: facing the challenges. Curr Opin Infect Dis 2007, 20:467-475.

2. Cowman AF, Baldi DL, Duraisingh M, Healer J, Mills KE, O'Donnell RA, Thompson J, Triglia T, Wickham ME, Crabb BS: Functional analysis of Plasmodium falciparum merozoite antigens: implications for erythrocyte invasion and vaccine development. Philos Trans R Soc Lond B Biol Sci 2002, 357:25-33.

3. Narum DL, Thomas AW: Differential localization of full-length and processed forms of PF83/AMA-I an apical membrane antigen of Plasmodium falciparum merozoites. Mol Biochem Parasitol 1994, 67:59-68.

4. Peterson MG, Marshall VM, Smythe JA, Crewther PE, Lew A, Silva A, Anders RF, Kemp DJ: Integral membrane protein located in the apical complex of Plasmodium falciparum. Mol Cell Biol 1989, 9:315I-3I54.

5. Kocken $\mathrm{CH}$, Withers-Martinez $\mathrm{C}$, Dubbeld MA, van der Wel A, Hackett F, Valderrama A, Blackman MJ, Thomas AW: High-level expression of the malaria blood-stage vaccine candidate Plasmodium falciparum apical membrane antigen $I$ and induction of antibodies that inhibit erythrocyte invasion. Infect Immun 2002, 70:447 I-4476.

6. Triglia T, Healer J, Caruana SR, Hodder AN, Anders RF, Crabb BS, Cowman AF: Apical membrane antigen I plays a central role in erythrocyte invasion by Plasmodium species. Mol Microbiol 2000, 38:706-718.

7. Stowers AW, Kennedy MC, Keegan BP, Saul A, Long CA, Miller LH: Vaccination of monkeys with recombinant Plasmodium falciparum apical membrane antigen I confers protection against blood-stage malaria. Infect Immun 2002, 70:696I-6967.

8. Nair M, Hinds MG, Coley AM, Hodder AN, Foley M, Anders RF, Norton RS: Structure of domain III of the blood-stage malaria vaccine candidate, Plasmodium falciparum apical membrane antigen I (AMAI). J Mol Biol 2002, 322:74I-753. 
9. Coley AM, Gupta A, Murphy VJ, Bai T, Kim H, Anders RF, Foley M, Batchelor AH: Structure of the malaria antigen AMAI in complex with a growth-inhibitory antibody. PLoS Pathog 2007, 3:1308-1319.

10. Lal AA, Hughes MA, Oliveira DA, Nelson C, Bloland PB, Oloo AJ, Hawley WE, Hightower AW, Nahlen BL, Udhayakumar V: Identification of T-cell determinants in natural immune responses to the Plasmodium falciparum apical membrane antigen (AMA-I) in an adult population exposed to malaria. Infect Immun 1996, 64:1054-1059.

II. Malkin EM, Diemert DJ, McArthur JH, Perreault JR, Miles AP, Giersing BK, Mullen GE, Orcutt A, Muratova O, Awkal M, Zhou H, Wang J, Stowers A, Long CA, Mahanty S, Miller LH, Saul A, Durbin AP: Phase I clinical trial of apical membrane antigen I: an asexual blood-stage vaccine for Plasmodium falciparum malaria. Infect Immun 2005, 73:3677-3685.

12. Cortes A, Mellombo M, Mueller I, Benet A, Reeder JC, Anders RF: Geographical structure of diversity and differences between symptomatic and asymptomatic infections for Plasmodium falciparum vaccine candidate AMAI. Infect Immun 2003, 71:1416-1426.

13. Escalante AA, Grebert HM, Chaiyaroj SC, Magris M, Biswas S, Nahlen $B L$, Lal AA: Polymorphism in the gene encoding the apical membrane antigen-I (AMA-I) of Plasmodium falciparum. X. Asembo Bay Cohort Project. Mol Biochem Parasitol 200I, I I 3:279-287.

14. Eisen DP, Marshall VM, Billman-Jacobe H, Coppel RL: A Plasmodium falciparum apical membrane antigen-I (AMA-I) gene apparently generated by intragenic recombination. Mol Biochem Parasitol 1999, 100:243-246.

15. Verra F, Hughes AL: Evidence for ancient balanced polymorphism at the Apical Membrane Antigen-I (AMA-I) locus of Plasmodium falciparum. Mol Biochem Parasitol 2000, 105:149-153.

16. Polley SD, Conway DJ: Strong diversifying selection on domains of the Plasmodium falciparum apical membrane antigen I gene. Genetics 200I, I58:I505-15I2.

17. Kennedy MC, Wang J, Zhang Y, Miles AP, Chitsaz F, Saul A, Long CA, Miller LH, Stowers AW: In vitro studies with recombinant Plasmodium falciparum apical membrane antigen I (AMAI): production and activity of an AMAI vaccine and generation of a multiallelic response. Infect Immun 2002, 70:6948-6960.

18. Polley SD, Mwangi T, Kocken CH, Thomas AW, Dutta S, Lanar DE, Remarque E, Ross A, Williams TN, Mwambingu G, Lowe B, Conway DJ, Marsh K: Human antibodies to recombinant protein constructs of Plasmodium falciparum Apical Membrane Antigen I (AMAI) and their associations with protection from malaria. Vaccine 2004, 23:7|8-728.

19. Vinayak S, Mittra P, Sharma YD: Wide variation in microsatellite sequences within each Pfcrt mutant haplotype. Mol Biochem Parasitol 2006, 147:101-108.

20. Nicholas KB, Nicholas HBj: GeneDoc: a tool for editing and annotating multiple sequence alignments. Distributed by the author 1997.

21. Rozas J, Sanchez-DelBarrio JC, Messeguer X, Rozas R: DnaSP, DNA polymorphism analyses by the coalescent and other methods. Bioinformatics 2003, 19:2496-2497.

22. Kumar S, Tamura K, Nei M: MEGA3: Integrated software for Molecular Evolutionary Genetics Analysis and sequence alignment. Briefings in Bioinformatics 2004, 5:.

23. Nei M, Gojobori T: Simple methods for estimating the numbers of synonymous and nonsynonymous nucleotide substitutions. Mol Biol Evol 1986, 3:4I8-426.

24. Tajima F: Statistical method for testing the neutral mutation hypothesis by DNA polymorphism. Genetics 1989, 123:585-595.

25. Escalante AA, Cornejo OE, Rojas A, Udhayakumar V, Lal AA: Assessing the effect of natural selection in malaria parasites. Trends Parasitol 2004, 20:388-395.

26. McDonald JH, Kreitman M: Adaptive protein evolution at the Adh locus in Drosophila. Nature 1991, 35 I:652-654.

27. Kimura M: A simple method for estimating evolutionary rates of base substitutions through comparative studies of nucleotide sequences. J Mol Evol 1980, 16:1 II-I20.

28. Polley SD, Chokejindachai W, Conway DJ: Allele frequency-based analyses robustly map sequence sites under balancing selec- tion in a malaria vaccine candidate antigen. Genetics 2003, 165:555-561.

29. Girard MP, Reed ZH, Friede M, Kieny MP: A review of human vaccine research and development: malaria. Vaccine 2007, 25: I567-I580.

30. Takala SL, Coulibaly D, Thera MA, Dicko A, Smith DL, Guindo AB, Kone AK, Traore K, Ouattara A, Djimde AA, Sehdev PS, Lyke KE, Diallo DA, Doumbo OK, Plowe CV: Dynamics of polymorphism in a malaria vaccine antigen at a vaccine-testing site in Mali. PLoS Med 2007, 4:e93.

31. Conway DJ: Natural selection on polymorphic malaria antigens and the search for a vaccine. Parasitol Today 1997, 13:26-29.

32. Hodder AN, Crewther PE, Matthew ML, Reid GE, Moritz RL, Simpson RJ, Anders RF: The disulfide bond structure of Plasmodium apical membrane antigen-I. J Biol Chem I 996, 27 I:29446-29452.

33. Sharma VP: Current scenario of malaria in India. Parassitologia 1999, $41: 349-353$.

34. Coley AM, Parisi K, Masciantonio R, Hoeck J, Casey JL, Murphy VJ, Harris KS, Batchelor AH, Anders RF, Foley M: The most polymorphic residue on Plasmodium falciparum apical membrane antigen I determines binding of an invasion-inhibitory antibody. Infect Immun 2006, 74:2628-2636.

35. Kocken $\mathrm{CH}$, Narum DL, Massougbodji A, Ayivi B, Dubbeld MA, van $\operatorname{der}$ Wel A, Conway DJ, Sanni A, Thomas AW: Molecular characterisation of Plasmodium reichenowi apical membrane antigen-I (AMA-I), comparison with P. falciparum AMA-I, and antibody-mediated inhibition of red cell invasion. Mol Biochem Parasitol 2000, 109: 147-156.

Publish with Bio Med Central and every scientist can read your work free of charge

"BioMed Central will be the most significant development for disseminating the results of biomedical research in our lifetime. "

Sir Paul Nurse, Cancer Research UK

Your research papers will be:

- available free of charge to the entire biomedical community

- peer reviewed and published immediately upon acceptance

- cited in PubMed and archived on PubMed Central

- yours - you keep the copyright 